\title{
Mucopolysaccharidosis: Cardiologic features and effects of enzyme-replacement therapy in 24 children with MPS I, II and VI
}

\author{
Marion M. M. G. Brands • Ingrid M. Frohn-Mulder • Marloes L. C. Hagemans • \\ Wim C. J. Hop • Esmee Oussoren • Wim A. Helbing • Ans T. van der Ploeg
}

Received: 1 November 2011 /Revised: 16 December 2011 / Accepted: 20 December 2011 / Published online: 26 January 2012

(C) The Author(s) 2012. This article is published with open access at Springerlink.com

\begin{abstract}
We determined the cardiologic features of children with MPS I, II and VI, and evaluated the effect of enzyme-replacement therapy (ERT) on cardiac disease. Twenty-four children aged 1-18 years with MPS I, II or VI were prospectively evaluated with echocardiogram and electrocardiogram from the start of enzyme-replacement therapy up to 6 years of treatment. At start of therapy, $66 \%$ had abnormal cardiac geometric features. Left-ventricular mass index (LVMI) was increased in half of the patients, due mainly to concentric hypertrophy in MPS I and II and to eccentric hypertrophy in MPS VI. Regurgitation was most severe in a subgroup of young MPS VI patients ( $<5$ years) at the mitral valve. At baseline, all patients had abnormal valves. The ECG showed no clear rhythm or conduction abnormalities; neither, in most patients, did it reflect the hypertrophy. After ERT, the LVMI Z-score normalized in
\end{abstract}

Communicated by: Ed Wraith

M. M. M. G. Brands · M. L. C. Hagemans · E. Oussoren •

A. T. van der Ploeg $(\varangle)$

Department of Pediatrics, division of Metabolic Diseases and

Genetics, Center for Lysosomal and Metabolic Diseases,

Erasmus MC University Medical Center Sophia's Children's

Hospital,

Dr. Molewaterplein 60,

3015 GJ Rotterdam, The Netherlands

e-mail: a.vanderploeg@erasmusmc.nl

I. M. Frohn-Mulder • W. A. Helbing

Department of Pediatrics, Division of Cardiology,

Erasmus MC - Sophia's Children's Hospital,

Rotterdam, the Netherlands

W. C. J. Hop

Department of Biostatistics, Erasmus MC,

Rotterdam, the Netherlands
$70 \%$ of the patients who had a Z-score $>2$. LVMI Z-scores decreased significantly in patients with MPS I and MPS II $(\mathrm{p}=0.04$ and $\mathrm{p}=0.032)$. Despite ERT, valve regurgitation increased in $60 \%$ of the patients. We conclude that all our MPS patients have cardiac abnormalities. The most severe cardiac disease was observed in a subgroup of young MPS VI patients. While ERT had an effect on LVMI and IVSd, it apparently had little or none on valve regurgitation.

\section{Introduction}

The mucopolysaccharidoses (MPS) are a group of lysosomal storage disorders, each caused by deficiency in one or more specific lysosomal enzymes involved in degrading glycosaminoglycans (Neufeld and Muenzer 2001). The resulting intralysosomal storage of glycosaminoglycans causes progressive disease that involves multiple organs, including the heart. All MPS are rare diseases; in the Netherlands their combined birth prevalence is estimated to be 1 in 22,000 (Poorthuis et al. 1999). Cardiac involvement has been reported for all forms of MPS, the most documented abnormalities being regurgitation, stenosis and morphologic changes of the cardiac valves, and cardiac hypertrophy (Dangel 1998; Gross et al. 1988; Rigante and Segni 2002; Wippermann et al. 1995). Recently, enzyme-replacement therapy (ERT) was introduced for MPS types I, II and VI. While this has alleviated several aspects of the disease, such as joint-mobility, endurance, and lung function (Harmatz et al. 2006; Muenzer et al. 2006; Wraith et al. 2007; Wraith et al. 2004), it is unclear whether it affects the cardiac abnormalities (Braunlin et al. 2006; Fesslova et al. 2009). In a cohort of patients with MPS I, II and VI we therefore studied these abnormalities before the start of ERT, and the effect of ERT upon them. 


\section{Materials and methods}

We prospectively studied 24 children with MPS I Hurler syndrome (OMIM 607014), MPS I Scheie syndrome (OMIM 607016), MPS II Hunter syndrome (OMIM 309900) and MPS VI Maroteaux-Lamy syndrome (OMIM 253200). The diagnosis of MPS was confirmed in all patients by mutation analysis and enzyme assay on leukocytes and fibroblasts. We subdivided the Hurler and Scheie patients on base of clinical presentation in combination with mutation anlaysis.

None of these patients had undergone hematopoietic stem cell transplantation. Patients with MPS I received $0.58 \mathrm{mg} /$ $\mathrm{kg} /$ week intravenous laronidase (Aldurazyme ${ }^{\circledR}$, Genzyme Corporation); MPS II patients received $0.5 \mathrm{mg} / \mathrm{kg} / \mathrm{week}$ intravenous idursulfase (Elaprase ${ }^{\circledR}$, Shire Pharmaceutical Inc); and MPS VI patients received $1.0 \mathrm{mg} / \mathrm{kg} /$ week intravenous galsulfase (Naglazyme ${ }^{\circledR}$, BioMarin Pharmaceutical Inc). The institutional review board approved the study and all patients provided written informed consent before participation.

A standardized assessment program was initiated to prospectively investigate cardiovascular abnormalities and function using a 12-lead electrocardiogram (ECG) and detailed echocardiogram. Cardiologic assessments were performed before the start of ERT and yearly thereafter, or more frequently if cardiologic abnormalities were severe.

Echocardiographic studies were performed by an experienced sonographer (JP) using a Philips iE33 xMAtrix Echocardiography System, Philips Medical Systems, Andover, MA, USA. Data were digitally stored and subsequently analyzed by two researchers (MB, IF). The following parameters were measured by 2D-guided M-mode tracing: end-diastolic left-ventricular internal dimension (LVIDd); inter-ventricular septum thickness in diastole (IVSd); leftventricular posterior wall thickness in diastole (LVPWd); and shortening fraction (SF). These values were compared with normal values according to Kampmann et al. (Kampmann et al. 2000). The left-ventricular mass index (LVMI) was calculated using the Devereux formula and indexed by body surface area with normal values according to Poutanen et al. (Poutanen and Jokinen 2007).

Diastolic filling was established using the E/A ratio by measuring mitral-inflow as determined by pattern-peak early filling (E) and late filling (A) velocities, and systolic function using the shortening fraction (Eidem et al. 2004). An E/A ratio $<1$ was considered abnormal. Specific attention was paid to the morphology and thickness of the valves, and also to valve regurgitation, which was determined according to the recommendations of the American Society of Echocardiography (Zoghbi et al. 2003). Trivial valve regurgitation was not considered abnormal.
The change over time in the thickness of the aortic and mitral valve was established during ERT, the first and last echocardiogram of every patient being studied by three experienced cardiologists and echocardiographists (IF, $\mathrm{MvO}, \mathrm{JP})$. The tricuspid and pulmononary valve could not be evaluated for changes in thickness over time since the imaging quality of these valves during follow-up was inconsistent. To evaluate the valves during ERT, one additional patient was excluded from the analysis, since his obesity impaired the echo window.

Due to the inability to lie quietly at time of the echocardiogram, there were no complete baseline echocardiograms of three patients (one MPS I Hurler and 2 MPS VI patients); for each, we therefore used an echocardiogram made before enzyme therapy as baseline value. The effects of enzyme therapy could be reliably evaluated in 20 patients, with a median follow-up of 104 weeks (range of treatment 48312 weeks of therapy). Four patients were excluded from the analysis of the effect of ERT: two had received ERT for less than one year; one had no reliable follow-up echocardiograms due to behavioural problems; and one had received a mitral graft which was considered to be a co-intervention bias.

Twelve-lead standard ECG's were recorded using a Mortara ELI 350, Mortara instrument Inc, Milwaukee, USA. ECGs were analyzed by hand by a single observer from a cardiologic core laboratory (Cardialysis BV). Pediatric reference values were obtained from Park et al. and corrected for age and heart rate. QT duration was corrected for heart rate using Bazett's formula: QTc $=$ QT $/ \sqrt{ }(\mathrm{RR}$ interval) (Park and Guntheroth 2006).

Every echocardiographic value obtained was transformed into a Z-score calculated as the difference between the observed value for the patient and the mean reference value divided by the standard deviation from the reference value. Z-scores $>2$ were considered abnormal. For every individual and at group level, change over time was analyzed using multiple linear regression with weeks of treatment as the independent variable. A p-value $<0.05$ was considered significant. All statistical analyses were conducted using SPSS version 15.0.

\section{Results}

Twenty-four patients were included in the study: 8 MPS I patients (5 Hurler and 3 Scheie), 6 MPS II patients, and 10 MPS VI patients. Fourteen patients (56\%) were Caucasian, 4 Arab (16\%), 4 Turkish (16\%), 1 Asian and 1 African. Most patients in the MPS VI group (70\%) were nonCaucasian. Table 1 shows the first and latest main clinical and cardiologic features of the echocardiograms per patient. All abnormal values $(\mathrm{Z}>2)$ are printed in bold. The median 


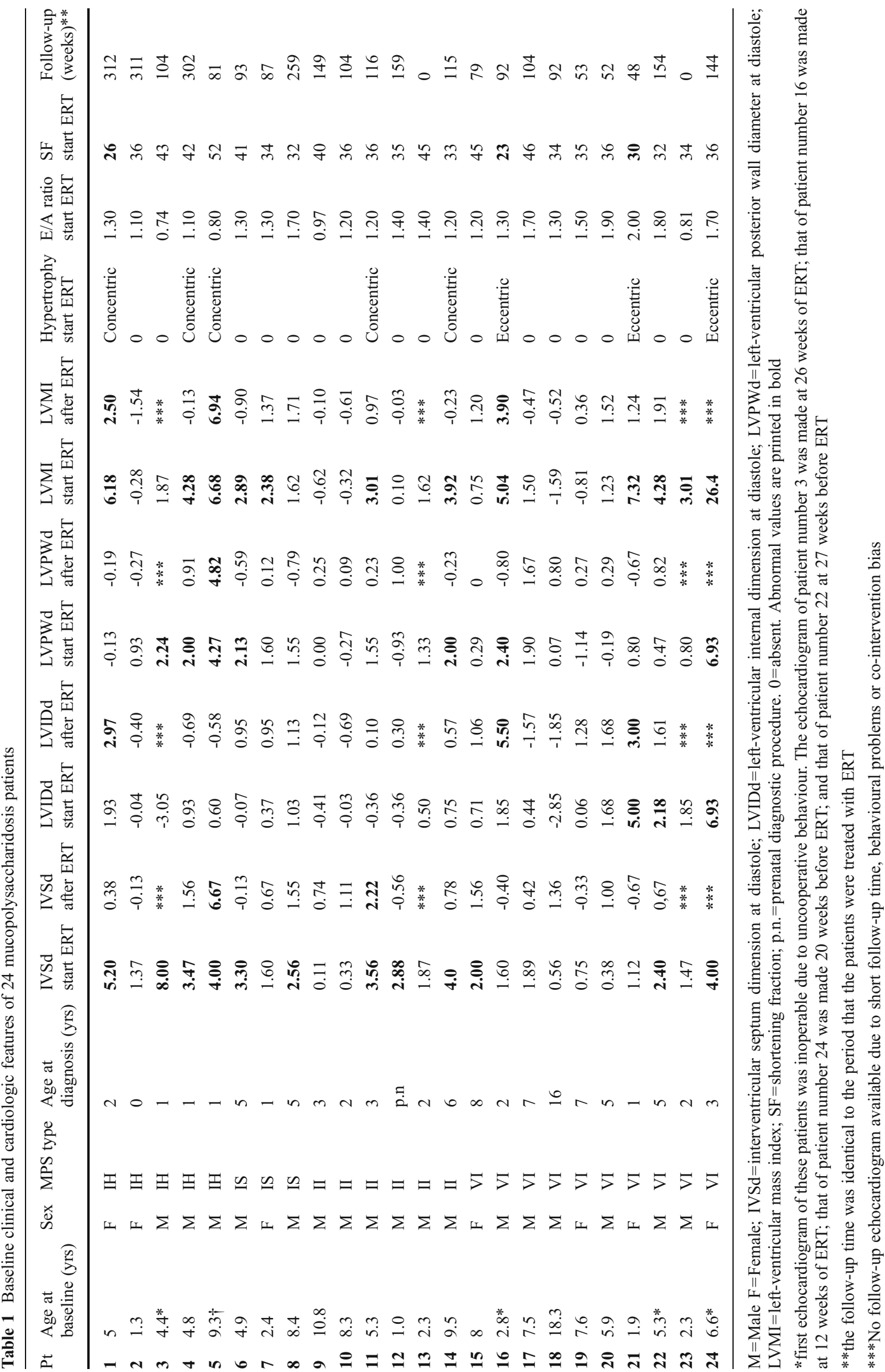


age at diagnosis was 2.5 years (range $0-16$ years); at start of therapy it was 5.3 years (range 1-18 years). Age ranged from 1.3-9.3 in the MPS I patient group, from 1.0-10.8 in the MPS II patient group and from 1.9-18.3 in the MPS VI patient group. In 3 patients, all of whom had MPS VI, the cardiac signs led to the diagnosis; these were also the only patients who were using cardiac medication (diuretics and ACE-inhibitors) at baseline. Due to respiratory failure after a respiratory infection, one MPS I Hurler patient treated with ERT died during follow-up at the age of 11 years.

At baseline, 16 of these 24 patients (66\%) had an abnormal Z-score for one of the geometric features (LVMI, LVPWd, IVSd, LVIDd), on echocardiogram. In MPS I and II, concentric hypertrophy was noted with an increase in IVSd and to a lesser extent an increase in LVPWd. In MPS VI, eccentric hypertrophy was observed in three of ten patients with related increased intraventricular dimensions. In one of these patients the shortening fraction was decreased. LVIDd was increased only in MPS VI patients.

The LVMI ranged from $57-268$ grams $/ \mathrm{m} 2$. Twelve of the 24 patients showed a LVMI Z-score $>2$. One MPS I Scheie patient had an increase in LVMI without any other increased parameters. Figure 1a and $\mathrm{b}$ shows an overview of
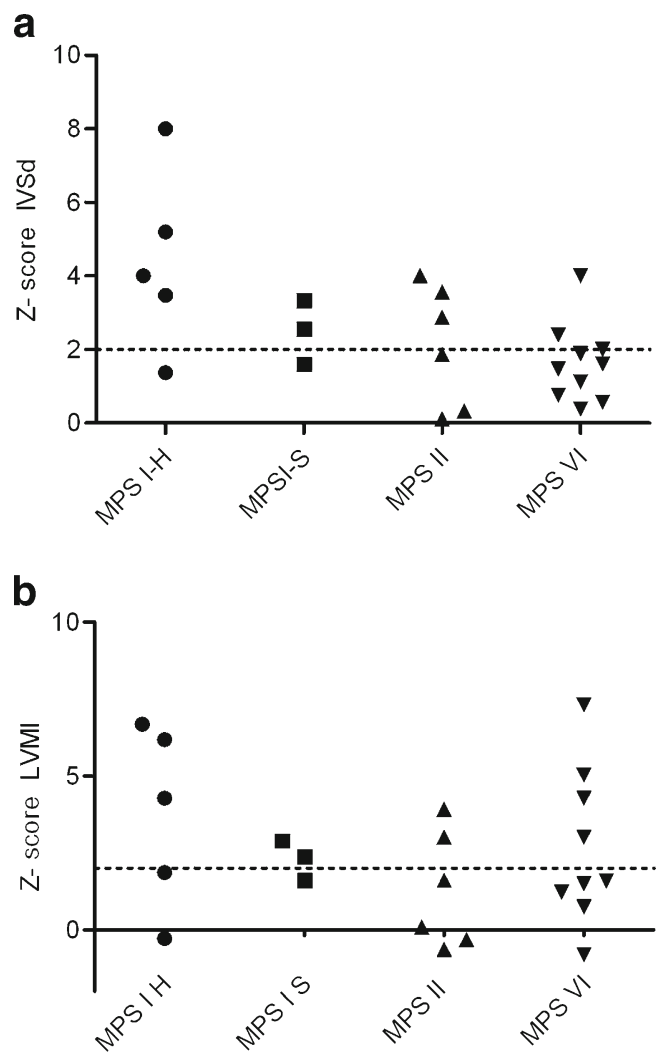

Fig. 1 a and b: Z-score for interventricular septum diameter in diastole (IVSd) and left-ventricular mass index (LVMI) in the different types of MPS. The dotted line represents the upper limit of the normal range $(=2)$. One patient (number 24) fell outside the axis range because she had an LVMI of 26.4 grams $/ \mathrm{m} 2$ the Z-scores of the LVMI and the IVSd of all patients by type of MPS.

At start of therapy, all patients had abnormal valves. An echocardiogram had been performed in one patient shortly after birth (at the age of 3 months) and 42 weeks before the start of enzyme-replacement therapy. In retrospect, it is now clear that slight echodense abnormalities were already visible in the mitral valve.

Fifteen patients $(63 \%)$ had a mild to severe regurgitation at one or more cardiac valves. The median age of this group was 5.3 years, whereas the median age of the nine patients without regurgitation was 2.4 years. Most regurgitation occurred at the mitral and aortic valve. There was regurgitation at the mitral valve in 7 patients (3 MPS I and 4 MPS VI), at the aortic valve in 7 patients (2 MPS I, 3 MPS II and 2 MPS $\mathrm{VI}$ ), and at the tricuspid valve and pulmonary valve in 2 patients (both MPS VI patients). The most severe regurgitation was in MPS VI patients. All four MPS VI patients with mitral valve regurgitation had been diagnosed at a young age (median 2.8 years); in two of them, MPS VI had been diagnosed after presentation with severe valve regurgitation. In one of these patient severe mitral regurgitation resulted in severe congestive heart failure. At 8 years old (one year after the start of enzyme therapy), she underwent a successful implantation of an artificial valve in mitral position and a valvuloplasty of the tricuspid valve. Five patients had a combination of aortic and mitral valve regurgitation: 1 MPS I Scheie patient, 3 MPS II patients, and 1 MPS VI patient.

Systolic function was impaired in one Hurler patient (FS 26\%) and two Maroteaux-Lamy patients (FS 30\% and FS 23\%). At baseline, the MPS VI patient who required mitral graft surgery had had a normal shortening fraction of $36 \%$. E/A ratio as a measure of diastolic function was abnormal in four patients. None of the patients had pulmonary hypertension.

Table 2 shows the baseline characteristics of the ECGs, $50 \%$ of which showed one or more abnormality. ECG abnormalities were generally of minor clinical importance.

The effects of enzyme therapy could be reliably evaluated in 20 patients (see Methods). At start of therapy, 10 patients had been younger than 5 years: 3 Hurler patients, 2 Scheie patient, 2 Hunter patients, and 3 Maroteaux-Lamy patients. Table 1 shows the first and latest results of the echocardiograms per patient.

A normal value of IVSd was achieved in 8 of the 10 patients with an IVSd Z-score above 2. At group level, the decrease in IVSd was significant for the group of MPS II patients $(-0.36 \mathrm{Z}$-score/year, $\mathrm{p}=0.05)$. In one MPS I patient the IVSd Z-score increased over 81 weeks of therapy. This patient died after 81 weeks of enzyme therapy.

The LVIDd decreased in one patient (MPS VI) with a Zscore for LVIDd above 2, but deteriorated in two patients 
Table 2 Baseline characteristics on electrocardiography in 24 MPS patients. MPS I $(\mathrm{H} / \mathrm{S})=$ MPS I Hurler and MPS I Scheie patients. $\mathrm{Bpm}=$ beats per minute

\begin{tabular}{lllll}
\hline & Baseline overall & MPS I (H/S) & MPS II & MPS VI \\
\hline ECG data & & & & \\
Rhythm & & & & \\
Sinus & $24(100 \%)$ & $8(100 \%)$ & $6(100 \%)$ & $10(100 \%)$ \\
Sinus tachycardia & $1(4 \%)$ & $1(13 \%)$ & $0(0 \%)$ & $0(0 \%)$ \\
Heart rate (bpm) & $107[78-155]$ & $120[100-155]$ & $118[80-150]$ & $93[78-125]$ \\
PR interval (ms) & $140[110,180]$ & $130[120,150]$ & $145[110,180]$ & $140[120,180]$ \\
Prolonged PR & $3(13 \%)$ & $0(0 \%)$ & $2(33 \%)$ & $1(10 \%)$ \\
QRS duration (ms) & $80[70,100]$ & $80[70,80]$ & $80[80-00]$ & $80[70,100]$ \\
Prolonged QRS & $3(13 \%)$ & $0(0 \%)$ & $2(\%)$ & $1(10 \%)$ \\
QTc interval (ms) & $415[378,453]$ & $402[390,417]$ & $440[412,453]$ & $422[378,441]$ \\
Prolonged QTc & $0(0 \%)$ & $0(0 \%)$ & $0(0 \%)$ & $0(0 \%)$ \\
Incomplete bundle branch block & $2(8 \%)$ & $0(0 \%)$ & $1(17 \%)$ & $1(10 \%)$ \\
Left fascicular block & 2 & $1(13 \%)$ & $0(0 \%)$ & $1(10 \%)$ \\
Left atrial enlargement & $2(8 \%)$ & $0(0 \%)$ & $1(17 \%)$ & $1(10 \%)$ \\
Right-ventricular hypertrophy & $1(4 \%)$ & $1(13 \%)$ & $0(0 \%)$ & $0(0 \%)$ \\
Left-ventricular hypertrophy & $1(4 \%)$ & $0(0 \%)$ & $0(0 \%)$ & $1(10 \%)$ \\
Repolarisation disorder & $2(8 \%)$ & $0(0 \%)$ & $1(17 \%)$ & $1(10 \%)$ \\
\hline
\end{tabular}

who had a normal diameter at baseline; in whom an increase in mitral regurgitation was likely to contribute to the increase in LVIDd.

LVPWd decreased in 4 of 5 patients with a Z-score above 2. The patient who did not respond on IVSd did not respond on LVPWd either.

Over time, Z-scores could be established reliably in 10 of the 12 patients who had had an LVMI Z-score above 2 at baseline. $Z$-scores normalized in 7 of these 10 patients. The decrease in Z-scores was statistically significant in the MPS I group $(-0.31$ $\mathrm{Z}$-score/year, $\mathrm{p}=0.04)$ and the MPS II group $(-0.26 \mathrm{Z}$-score/ year, $p=0.032$ ). In 4 individual MPS I and VI patients the decrease in Z-score was significant with values ranging from 0.002 to 0.043 . Figure 2 shows their regression lines.

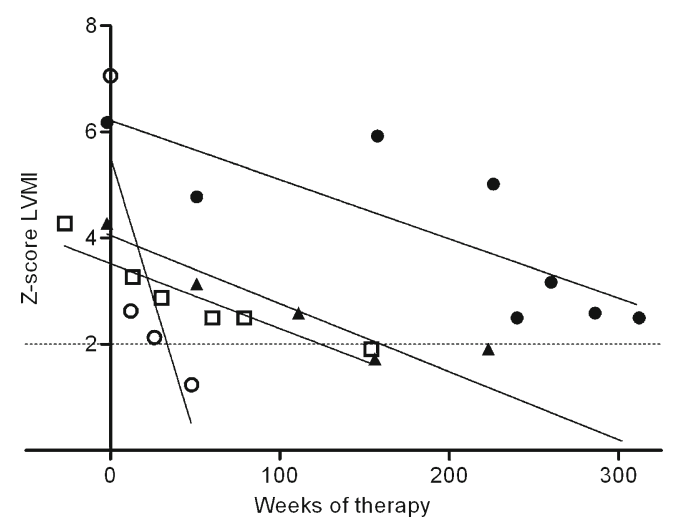

Fig. 2 Regression lines of patients with significant improvement in left-ventricular mass index (LVMI). Z-score LVMI = Z-score of the left-ventricular mass index. $\bullet=$ patient number one $(\mathrm{p}=0.015) ; \boldsymbol{\Delta}=$ patient number four $(\mathrm{p}=0.002) ; \circ=$ patient number $21(\mathrm{p}=0.009) ; \square=$ patient number $22(\mathrm{p}=0.043)$
Regurgitation increased after start of ERT in $60 \%$ of the patients and was mostly, in $40 \%$ of the cases, seen at the mitral valve. In most cases regurgitation increased from none to mild. In one MPS VI patient mitral valve regurgitation increased from moderate to severe; aortic regurgitation increased from mild to moderate in two patients, one MPS VI and one MPS I patient. In $15 \%$ of the patients an improvement in regurgitation was observed. Patients with moderate to severe regurgitation did not ameliorate.

Thickness of the mitral valve decreased in $6 / 20$ patients (1 MPS IS, 2 MPS II and 3 MPS VI), who had been treated with enzyme therapy for a median of 86 weeks (range of 52159 weeks). Eight $/ 20$ patients had an increase in mitral valve thickness (3 MPS I H, 1 MPS I S, 2 MPS II and 2 MPS VI); three of these patients also had an increase in aortic valve thickness, and had been treated for a median of 110 weeks (range of 48-312 weeks).

The thickness of the aortic valve decreased in 5 patients (2 Hunter and 3 Maroteaux-Lamy patients, range of therapy 52-159 weeks) and increased in three. There was no clear relationship between the increase in thickness of the valves and the development of regurgitation during ERT. three patients (FS 35\% and 39\% at last assessment in patients 16 and 21), but remained abnormal in the third (patient 1). The same patients with an impaired diastolic function at start of therapy continued to have an E/A ratio $<1$ at follow-up, except for one patient.

The three MPS VI patients which used medication at start of therapy remained on medication during the course of ERT. The dosage could be reduced in one patient, and the
Left ventricular shortening fraction normalized in two of 
medication regiment was changed in one other patient who received a mitral graft.

No clinically important changes were found at the follow-up ECGs. We report only the changes found at follow-up. One patient had atrial beats on the follow-up electrocardiograms. The increased PR-interval present at baseline in 2 MPS II patients was absent later. The QRScomplex increased in duration in the patient who already had a prolonged QRS at baseline. This was probably due to the left-ventricular hypertrophy, which was also seen on the follow-up echocardiogram. Hypertrophy disappeared on ECG in the only patient who had shown signs of hypertrophy on ECG at baseline; this was consistent with the findings on echocardiogram.

\section{Discussion}

We studied cardiac abnormalities and the effects of enzyme therapy in children with MPS I, II and VI. As the current literature points out, cardiologic abnormalities are a common finding in patients with MPS (Dangel 1998; Gross et al. 1988; Rigante and Segni 2002; Wippermann et al. 1995; Fesslova et al. 2009; Leal et al. 2010; Mohan et al. 2002; John et al. 1990). At start of therapy, all our patients had cardiologic abnormalities, which is remarkable, as they were all relatively young.

At start of therapy, $66 \%$ had abnormal cardiac geometric features caused either by increased cardiac-wall diameter (IVSd) or by cardiac dilatation. Interestingly, while most MPS I and II patients had hypertrophic cardiomyopathy findings that are consistent with studies reporting the occurrence of increased IVSd and left-ventricular hypertrophy in MPS I and II patients (Dangel 1998; Mohan et al. 2002) eccentric hypertrophy was seen solely in the patients with MPS VI. Reports of ventricular dilatation in MPS VI are still limited (Leal et al. 2010; Fong et al. 1987; Golda et al. 2011). Dilatation in our MPS VI patient group was explained mainly by mitral-valve regurgitation. Primary regurgitation causes the left-ventricular remodeling and dilatation. Generally in MPS, we hypothesize that the increase in geometric features is partly due to the compensatory mechanism caused by the mitral regurgitation and partly because of the accumulation of storage material. The asymmetric hypertrophy in the wall diameters with an increased IVSd in MPS I and MPS II and an increased LVIDd in MPS VI indicates this.

While heparan sulphate and dermatan sulphate accumulate in MPS I and II, storage in MPS VI is confined to dermatan sulphate. Leal et al. hypothesized that storage of dermatan sulphate is the main cause of valve deformation and dysfunction in MPS (Leal et al. 2010). We in turn hypothesize that storage of heparan sulphate is an additional cause of increased thickness of the walls, particularly in the septum, while the storage of dermatan sulphate leads to valve impairment and consequent eccentric hypertrophy.

The presence of affected valves in all our patients from the age of 3 months shows that the deposition of glycosaminoglycans in the heart can occur at a very early age, and may already start in utero; a similar conclusion was drawn from studies in MPS VII mice (Vogler et al. 2005). The mitral and aortic valves were the most affected, which is consistent with earlier reports (Rigante and Segni 2002; Wippermann et al. 1995). There was no clear relationship between thickened valves and regurgitation.

Although the most severe valve regurgitation and cardiac disease occurred in the group of MPS VI patients, this group also had the least cardiac involvement. Apparently these patients can be subdivided into two categories: one in which patients present before the age of 5 years, with cardiac problems as the main presenting feature; and another in which clinical features such as joint pains and mobility problems lead to the diagnosis later in life. While the literature has concluded that the severity of cardiologic abnormalities in MPS VI is either minor (Fesslova et al. 2009; Mohan et al. 2002) or severe (Dangel 1998; Wippermann et al. 1995), our results support both conclusions. These differences may be explained by further genotype-phenotype studies (Jurecka et al. 2011).

Although 50\% of ECGs showed abnormalities, none showed clinically important rhythm or conduction abnormalities. Generally, ECGs did not reflect the hypertrophy found in patients, possibly because glycosaminoglycans have a low electric conductance (Rigante and Segni 2002; Nelson et al. 1990). We therefore conclude that ECG is not a reliable tool for identifying cardiologic abnormalities in MPS, and that echocardiography should be mandatory in all patients. Although conduction abnormalities were not found in our group of children with MPS, they may occur at later age (Braunlin et al. 2011). Therefore ECG should remain part of the follow-up program of MPS patients.

There have been few reports on the effects of enzymereplacement therapy on cardiologic abnormalities in MPS (Braunlin et al. 2006; Fesslova et al. 2009; Scarpa et al. 2009; Kakkis et al. 2001). After ERT, the Z-score normalized in $70 \%$ of our patients who had had a $Z$-score $>2$ for LVMI; in four patients this was significant. This suggests that ERT has some effect on GAG accumulation in cardiac tissue. Earlier Wraith et al., Braunlin et al. and Okuyama et al. reported positive or mildly positive effects on the cardiac diameters in MPS I and II patients (Wraith et al. 2007; Braunlin et al. 2006; Okuyama et al. 2010).

Effects on cardiac valve regurgitation seem to be limited. In $60 \%$ of patients, valve regurgitation increased, and mitral valve regurgitation increased in $40 \%$. On the other hand, $15 \%$ of patients showed minor improvements in mitral valve regurgitation. There was no clear relationship between the 
increase in thickness of the valves and the development of regurgitation during ERT. In some patients, the thickness of the valves decreased, while in others it increased or stabilized. We should note, however, that 2D imaging is not the best way of assessing morphologic change. For assessment of valve morphology, 3D echo currently is the technique of choice.

Although it is unknown whether the percentage of patients with dysmorphology and deterioration of valve regurgitation is similar to that of patients not receiving ERT (Fesslova et al. 2009; Mohan et al. 2002), our findings suggest overall that the cardiac valves are only slightly accessible to ERT. This might be explained by the ultra structure of the cardiac valves. While microvasculature can be present in the heart valves, the myofiboblasts composing the valves are supplied with oxygen mainly by diffusion from the valve surface (Misfeld and Sievers 2007) and therefore not easy targets for the relatively large therapeutic enzymes that need to enter the cells via receptor mediated endocytosis. As a consequence, the correction of MPS storage in the heart valves remains challenging. While other studies agree that ERT has a limited effect on the cardiac valves in MPS I and MPS II (Fesslova et al. 2009), Scarpa et al. described an improvement in valve disease in one MPS VI patient in an Italian cohort (Scarpa et al. 2009).

In our group, 1 MPS I Hurler patient died after a respiratory infection during follow-up. This, notably, was one of the few patients in whom ERT had no effect on any of the cardiologic parameters. Further research is required to investigate the role of prognostic factors.

In conclusion, all MPS I, II and VI patients included in our study had abnormalities of the valves, $63 \%$ had valve regurgitation, and $66 \%$ had one or more abnormal left ventricular geometric features at an early age. While ERT seemed to have little or no effect on valve regurgitation, it had some effect on cardiac dimensions. Cardiac disease was most severe in a subset of young MPS VI patients.

Acknowledgments We would like to thank the families who traveled across the Netherlands with their children for several evaluations at the Center for Lysosomal and Metabolic Disorders; J. Ponsen (JP) for her echocardiographic work; M. van Osch-Gevers (MvO) pediatric cardiologist for her evaluation of the cardiac valves; J. Hardon and $\mathrm{H}$. Nelisse for their contribution to the study as research nurse and A.J.J. Reuser and D. Alexander for critical review of the manuscript.

Details of funding The Research on MPS at Erasmus MC is financially supported by the European Union, $7^{\text {th }}$ Framework Programme 'Euclyd - a European Consortium for Lysosomal Storage Diseases' [health F2/2008 grant agreement 201678], ZonMw - Dutch organization for healthcare research and innovation of care [Grant 152001003 and 152001004] and TI Pharma initiative 'Sustainable Orphan Drug Development through Registries and Monitoring (T6-208)'. The authors confirm independence from the sponsors; the content of the article has not been influenced by the sponsors.

Open Access This article is distributed under the terms of the Creative Commons Attribution Noncommercial License which permits any noncommercial use, distribution, and reproduction in any medium, provided the original author(s) and source are credited.

\section{References}

Braunlin EA, Berry JM, Whitley CB (2006) Cardiac findings after enzyme replacement therapy for mucopolysaccharidosis type I. Am J Cardiol 98:416-418

Braunlin EA, Harmatz PR, Scarpa M et al (2011) Cardiac disease in patients with mucopolysaccharidosis: presentation, diagnosis and management. J Inherit Metab Dis

Dangel JH (1998) Cardiovascular changes in children with mucopolysaccharide storage diseases and related disorders-clinical and echocardiographic findings in 64 patients. Eur J Pediatr 157:534-538

Eidem BW, McMahon CJ, Cohen RR et al (2004) Impact of cardiac growth on Doppler tissue imaging velocities: a study in healthy children. J Am Soc Echocardiogr 17:212-221

Fesslova V, Corti P, Sersale G et al (2009) The natural course and the impact of therapies of cardiac involvement in the mucopolysaccharidoses. Cardiol Young 19:170-178

Fong LV, Menahem S, Wraith JE, Chow CW (1987) Endocardial fibroelastosis in mucopolysaccharidosis type VI. Clin Cardiol 10:362-364

Golda A, Jurecka A, Tylki-Szymanska A (2011) Cardiovascular manifestations of mucopolysaccharidosis type VI (Maroteaux-Lamy syndrome). Int J Cardiol Jul 5. [Epub ahead of print]

Gross DM, Williams JC, Caprioli C, Dominguez B, Howell RR (1988) Echocardiographic abnormalities in the mucopolysaccharide storage diseases. Am J Cardiol 61:170-176

Harmatz P, Giugliani R, Schwartz I et al (2006) Enzyme replacement therapy for mucopolysaccharidosis VI: a phase 3, randomized, double-blind, placebo-controlled, multinational study of recombinant human $\mathrm{N}$-acetylgalactosamine 4-sulfatase (recombinant human arylsulfatase B or rhASB) and follow-on, open-label extension study. J Pediatr 148:533-539

John RM, Hunter D, Swanton RH (1990) Echocardiographic abnormalities in type IV mucopolysaccharidosis. Arch Dis Child 65:746-749

Jurecka A, Golda A, Opoka-Winiarska V, Piotrowska E, TylkiSzymanska A (2011) Mucopolysaccharidosis Type VI (MaroteauxLamy syndrome) with a predominantly cardiac phenotype. Mol Genet Metab

Kakkis ED, Muenzer J, Tiller GE et al (2001) Enzyme-replacement therapy in mucopolysaccharidosis I. N Engl J Med 344:182188

Kampmann C, Wiethoff CM, Wenzel A et al (2000) Normal values of $\mathrm{M}$ mode echocardiographic measurements of more than 2000 healthy infants and children in central Europe. Heart 83:667-672

Leal GN, de Paula AC, Leone C, Kim CA (2010) Echocardiographic study of paediatric patients with mucopolysaccharidosis. Cardiol Young 20:254-261

Misfeld M, Sievers HH (2007) Heart valve macro- and microstructure. Philos Trans R Soc Lond B Biol Sci 362:1421-1436

Mohan UR, Hay AA, Cleary MA, Wraith JE, Patel RG (2002) Cardiovascular changes in children with mucopolysaccharide disorders. Acta Paediatr 91:799-804 
Muenzer J, Wraith JE, Beck M et al (2006) A phase II/III clinical study of enzyme replacement therapy with idursulfase in mucopolysaccharidosis II (Hunter syndrome). Genet Med 8:465-473

Nelson J, Shields MD, Mulholland HC (1990) Cardiovascular studies in the mucopolysaccharidoses. J Med Genet 27:94-100

Neufeld EF, Muenzer J (2001) The Mucopolysaccharidosis. In: Scriver CR, Beadet AL, Valle D, Childs B, Kinzler KW, Vogelstein B (eds) The metabolic and molecular bases of inherited disease. McGraw-Hill Professional, New York, pp 3421-3452

Okuyama T, Tanaka A, Suzuki Y et al (2010) Japan Elaprase Treatment (JET) study: idursulfase enzyme replacement therapy in adult patients with attenuated Hunter syndrome (Mucopolysaccharidosis II, MPS II). Mol Genet Metab 99:18-25

Park MK, Guntheroth WG (2006) How to read pediatric ECGs. Elsevier, Dordrecht

Poorthuis BJ, Wevers RA, Kleijer WJ et al (1999) The frequency of lysosomal storage diseases in the Netherlands. Hum Genet 105:151156

Poutanen T, Jokinen E (2007) Left ventricular mass in 169 healthy children and young adults assessed by three-dimensional echocardiography. Pediatr Cardiol 28:201-207

Rigante D, Segni G (2002) Cardiac structural involvement in mucopolysaccharidoses. Cardiology 98:18-20
Scarpa M, Barone R, Fiumara A et al (2009) Mucopolysaccharidosis VI: the Italian experience. Eur J Pediatr 168:1203-1206

Vogler C, Levy B, Galvin N, Lessard M, Soper B, Barker J (2005) Early onset of lysosomal storage disease in a murine model of mucopolysaccharidosis type VII: undegraded substrate accumulates in many tissues in the fetus and very young MPS VII mouse. Pediatr Dev Pathol 8:453-462

Wippermann CF, Beck M, Schranz D, Huth R, Michel-Behnke I, Jungst BK (1995) Mitral and aortic regurgitation in 84 patients with mucopolysaccharidoses. Eur J Pediatr 154:98-101

Wraith JE, Clarke LA, Beck M et al (2004) Enzyme replacement therapy for mucopolysaccharidosis I: a randomized, doubleblinded, placebo-controlled, multinational study of recombinant human alpha-L-iduronidase (laronidase). J Pediatr 144:581-588

Wraith JE, Beck M, Lane R et al (2007) Enzyme replacement therapy in patients who have mucopolysaccharidosis I and are younger than 5 years: results of a multinational study of recombinant human alpha-L-iduronidase (laronidase). Pediatrics 120:e37-e46

Zoghbi WA, Enriquez-Sarano M, Foster E et al (2003) Recommendations for evaluation of the severity of native valvular regurgitation with two-dimensional and Doppler echocardiography. J Am Soc Echocardiogr 16:777-802 